\title{
Ovarian Cancer by FIGO Stage
}

National Cancer Institute

\section{Source}

National Cancer Institute. Ovarian Cancer by FIGO Stage. NCI Thesaurus. Code C128081.

A category of staging terms for ovarian cancer according to the International Federation of Gynecology and Obstetrics (FIGO), 2014. AJCC ovarian cancer stage terms from the 6th and 7th editions that are synonymous to the FIGO ovarian cancer staging classification of 2014 are included as preferred terms. 